\title{
A new device for beating heart bipolar radiofrequency atrial ablation
}

\author{
Gianluca Bonanomi, MD \\ David Schwartzman, MD \\ David Francischelli, MS ${ }^{\mathrm{c}}$ \\ Kim Hebsgaard, MD \\ Marco A. Zenati, MD ${ }^{a, b}$
}

Objective: A technique for mimicking left atrial atriotomies using an ablation device that can be deployed without cardiopulmonary bypass has been developed.

Methods: In 12 healthy large $(35-50 \mathrm{~kg})$ adult pigs, maze-like ablation lesions were directly applied to the left atrial epicardium on the beating heart. The ablation device is irrigated, with a bipolar "hemostat" morphology, utilizing radiofrequency energy. Prior to and after ablation, left atrial electromechanical properties were measured during sinus rhythm in the latest 5 pigs using percutaneous endocardial catheter electromechanical mapping and intracardiac echocardiography. Pathologic analysis was performed acutely.

Results: All ablation lesions demonstrated conduction block along their entire course. Global left atrial conduction time (49.4 \pm 8.8 milliseconds before vs $58.8 \pm$ 9 milliseconds after) and pattern were not significantly altered. Although a significant amount $(17.12 \% \pm 9 \%)$ of myocardium was either ablated or electrically isolated, ablation was not associated with significant alterations in global left atrial mechanics (left atrium ejection fraction $19 \%$ before vs $17 \%$ after; pulmonary vein peak flow velocity $1.22 \mathrm{~m} / \mathrm{s}$ before vs $1.38 \mathrm{~m} / \mathrm{s}$ after; peak mitral inflow velocity $2.34 \mathrm{~m} / \mathrm{s}$ before vs $2.64 \mathrm{~m} / \mathrm{s}$ after), mitral valve function, nor left ventricular function. There was no evidence of atrial thrombus formation. Transmurality was achieved in most lesions with no evidence of charring or barotrauma.

Conclusions: Utilizing this ablation device, atrial lesions similar to the left component of the Maze procedure were deployed with uniform success in a beating heart without cardiopulmonary bypass or atriotomy and without adverse effects on left atrial electromechanics.

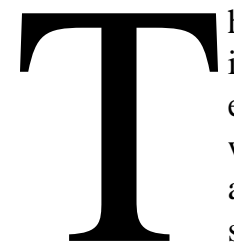

he surgical approach to atrial fibrillation (AF) is evolving since the introduction of the maze procedure by Cox and associates. ${ }^{1}$ Less extensive atrial incisions, replacement of the cut and sew lesions with cryoablation, and microwave or radiofrequency (RF) energy application and beating heart approaches have already been described although further studies are needed to confirm their safety and efficacy. ${ }^{2-5}$ A new device has been developed to create surgical ablation of the atrium from the epicardial surface of the heart using RF energy. The ablation device features a "hemostat" morphology able to deliver bipolar RF energy and is irrigated. The purpose of this study was to investigate the feasibility, safety, and reproducibility of an anatomy-based ablation of the left atrium (LA) that was performed on the epicardial surface of the beating heart in a healthy swine model. Moreover, an electromechanical assessment of the LA was performed prior to and after ablation using percutaneous endocardial catheter mapping and intracardiac echocardiography. Such a procedure delivered without cardiopulmonary bypass (CPB) would, if 


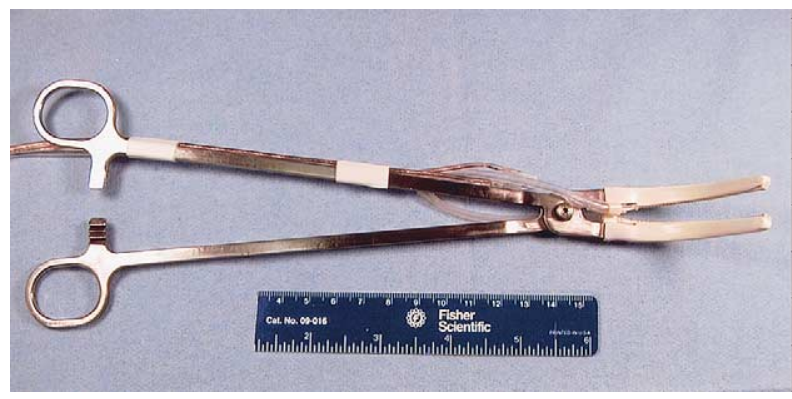

Figure 1. The ablation device featuring the bipolar delivery of RF energy and saline irrigation on each arm of a clamping instrument.

uniformly safe and if similar outcomes to the maze procedure are realized, become applicable to a broad population of patients with or at risk for $\mathrm{AF}$ who are undergoing cardiac surgery and for a smaller population as the lone indication for surgery.

\section{Materials and Methods Animals}

Twelve healthy Hanford swine of either sex were used in this study. The mean weight was $40.4 \pm 5.6 \mathrm{~kg}$. All animals received humane care in a facility fully accredited by the Council on Accreditation of the Association for Assessment and Accreditation of Laboratory Animal Care, in accordance with the Guide for the Care and Use of Laboratory Animals published by the National Institute of Health (NIH publication no. 85-23, revised 1985). The study protocol was approved by the Institutional Animal Care and Use Committee of the University of Pittsburgh.

\section{Anesthesia}

Animals were premedicated with intramuscular ketamine $(20 \mathrm{mg} /$ $\mathrm{kg}$ ) and isoflurane (1\%-2\%) delivered by face mask prior to endotracheal intubation. Anesthesia was maintained with isoflurane mixed with low flow oxygen and room air. Vascular access was obtained by surgical cut-down of the right carotid artery for blood pressure monitoring and the right jugular vein for fluid administration. Mean arterial blood pressure was kept stable around $75 \mathrm{~mm} \mathrm{Hg}$. At the completion of the experiments, the animals were put to death using a lethal injection of potassium chloride while under anesthesia.

\section{Radiofrequency Ablation}

RF energy was delivered to the heart using the prototype of a new ablation device manufactured by Medtronic Inc. The system was designed to ablate tissue by the induction of thermal necrosis in the targeted tissues. The surgical hand piece incorporates 2 electrodes on separate arms of a hemostat instrument able to deliver bipolar RF energy (Figure 1). Each electrode features a platinum/iridium conductor, a porous polymer, and saline irrigation $(250 \mathrm{~mL} / \mathrm{h})$. Power (35 watts) was delivered from a Cardioblate Generator (Medtronic, Inc) connected to a custom LabView (National Instruments, Austin, Tex) software that monitors tissue impedance, in real time, every 200 milliseconds as a measure of lesion transmu-

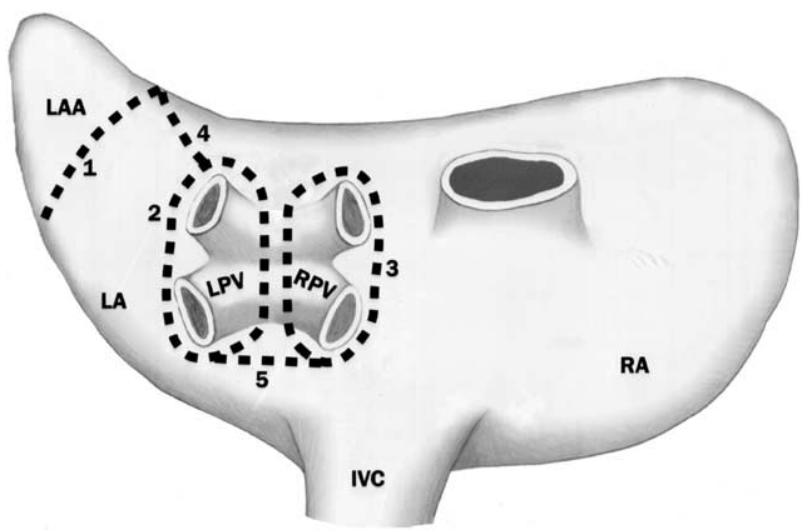

Figure 2. Epicardial atrial ablation pattern. $L A A$, Left atrial appendage; $L A$, left atrium; $R A$, right atrium; $L P V$, left pulmonary vein; $R P V$, right pulmonary vein; $I V C$, inferior vena cava; dotted lines, ablation lesions.

rality. The temperature was not measured at the probe, and the saline irrigation for cooling of the electrode-tissue interface allowed us to delay the production of microbubbles and a sudden rise in impedance that could prevent the device from applying sufficient energy to the tissue.

\section{Surgical Procedure}

The heart was exposed through a median sternotomy and a beating heart procedure in which the RF energy was applied to the LA from the epicardial surface was performed. The Starfish (Medtronic Inc) heart positioning system was applied to the apex of the left ventricle and the heart lifted to expose the posterior wall of the LA. The left and right pulmonary veins (LPV and RPV) as well as the inferior vena cava were surgically isolated to enable the placement of the ablation device. A long right-angle tool was used to dissect the common trunk of the LPV in proximity of the LA wall. Likewise, the common trunk of the RPV was isolated at the junction with the LA by entering and dissecting the Waterston groove. A modification of the left-sided maze was delivered with the RF device as illustrated in Figure 2. The procedure included lesions to isolate the LPV, the RPV, and the left atrial appendage (LAA) as well as connecting lesions between the two pulmonary veins and between the LPV and the LAA. The connecting lesion between the LPV and the RPV was performed on the inferior aspect of the LA. In preliminary experiments, access to the roof of the LA to deliver a superior connecting lesion between the LPV and the RPV was attempted but the technique proved to be more demanding and resulted in poor transmurality.

\section{Lesion Assessment}

The real-time assessment of lesion creation was performed by monitoring the decrease in impedance with respect to time. The transmurality algorithm did not operate on absolute impedance readings but rather on relative changes in tissue impedance during the ablation cycle. Heating tissue caused denaturation of the sodium potassium pumps within the cell wall, leading to a decrease in tissue impedance. By continually calculating the change in 


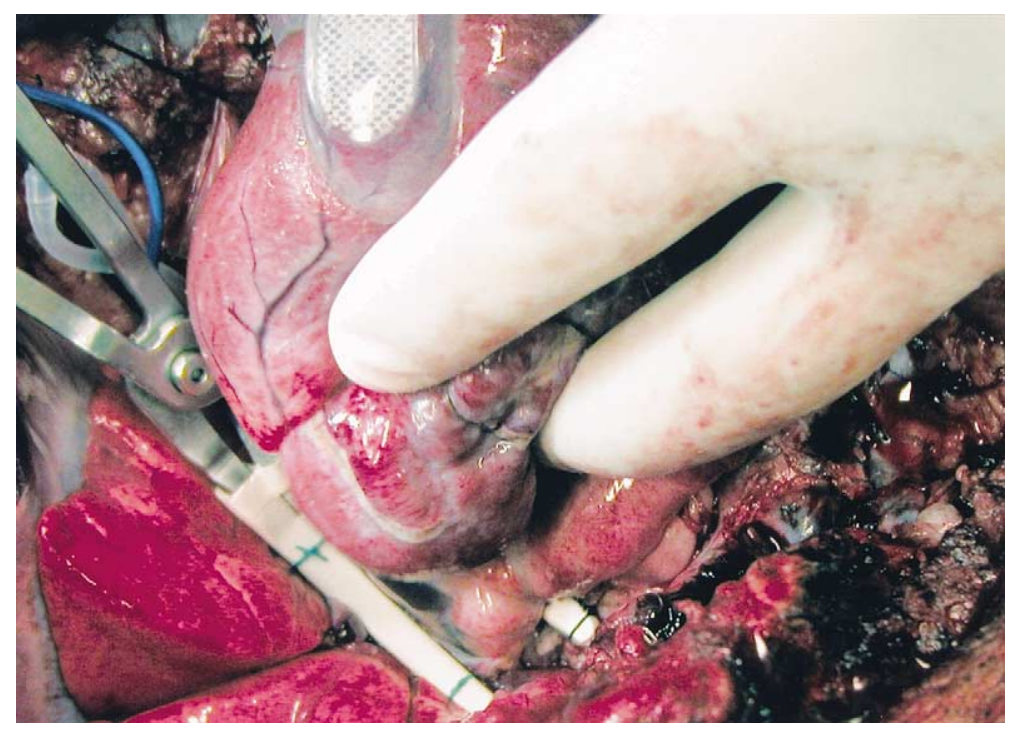

Figure 3. The left pulmonary vein is isolated following minimal surgical dissection and the RF energy delivered using the bipolar device.

impedance the tissue was ablated until there was a plateau in the impedance that was considered as the point of transmurality. Therefore, this algorithm was independent of tissue type or location. Following the lesion creation, the assessment of the efficacy of ablation to produce conduction block was accomplished through an attempt to pace across each lesion. Conduction block was considered as the inability to capture the atrium at $15 \mathrm{~mA}$.

\section{Intracardiac Echocardiography}

Vascular access was achieved via percutaneous right and left femoral venipuncture using the Seldinger technique. The Acunav intracardiac echocardiography catheter (Acuson Inc, Mountain View, Calif) was advanced into the right atrium for high-quality imaging of both atria, including B-mode imaging as well as pulsed, continuous wave and color Doppler at 7.5 or $8.5 \mathrm{MHz}{ }^{6}$ A qualitative and quantitative evaluation of the heart was performed just prior to and 60 minutes after atrial ablation in 5 pigs. The heart was examined for any evidence of thrombus formation and wall motion abnormality (WMA). RPV and LPV diameter and flow as well as peak transmitral valve inflow (TMV) and left ventricle ejection fraction (LVEF) were assessed. For pulmonary vein flow the peak systolic (P1), diastolic (P2), and retrograde (A) waves were measured as previously described. ${ }^{7}$

\section{Electromechanical Mapping}

Following intracardiac echocardiography, an electromechanical study was performed before and after epicardial atrial ablation in 5 pigs. A mapping system (NOGA; Biosense/Webster, Diamond Bar, Calif) utilizing synchronous extracorporeal magnetic fields to determine the location and orientation of a sensor mounted within a 7F intracardiac catheter (Navi-Star; Biosense Webster, Diamond Bar, Calif) provided detailed activation maps as well as local electrocardiogram data. ${ }^{8}$ A reference catheter was placed on the animal's back. The LA was entered via atrial transseptal puncture to enable the advancement of the mapping catheter. The method samples the location of the intracardiac catheter throughout the cardiac cycle at different endocardial sites and reconstructs a dynamic tridimensional (3D) electromechanical map of the LA. Local activation time and unipolar and bipolar electrical potentials are acquired simultaneously, color-coded, and represented on the 3D anatomical map. From the electromechanical information during systole and diastole the system can provide global and regional functional data including the LA end-diastolic and end-systolic volumes (LA ED and LA ES), the LA ejection fraction (LA EF), the electrical activation time (AT) and bipolar endocardial electrical signals.

\section{Postmortem Analysis}

After each pig was killed, the chest was carefully inspected to exclude any damage to cardiac and mediastinal structures; the heart was excised and analyzed macroscopically for any evidence of trauma or thrombus formation in the LA. The specimen was stained with $1 \%$ triphenyltetrazolium chloride (TTC) and cross sections encompassing the lesions were analyzed for dimensions and transmurality.

\section{Statistical Analysis}

Results are reported as mean and standard deviation values. For the comparison of preablation and postablation variables a paired Student $t$ test was used. A $P$ value $<.05$ was considered significant.

\section{Results}

\section{Surgical Ablation}

The hand piece design enabled the delivery of the encircling lesions around the LPV and the RPV following minimal surgical dissection (Figure 3). The LAA lesion was delivered by clamping the finger-shaped appendage at its base. The hemostat design was suitable for delivering the inter- 
TABLE 1. Ablation lesion features

\begin{tabular}{|c|c|c|c|c|c|}
\hline Lesion & $\begin{array}{l}\text { Energy } \\
\text { (watts) }\end{array}$ & $\begin{array}{l}\text { Ablation time } \\
\text { (seconds) }\end{array}$ & $\begin{array}{l}\text { Threshold } \\
\text { (mA) }\end{array}$ & $\begin{array}{l}\text { Transmurality } \\
\text { (\% cases; n) }\end{array}$ & $\begin{array}{l}\text { Width } \\
\text { (mm) }\end{array}$ \\
\hline LAA & 35 & $30 \pm 7$ & $>20$ (15 in 2 cases) & $100 \% ; 12$ & $3 \pm 0.5$ \\
\hline LPV & 35 & $20 \pm 5$ & $>20$ & $100 \% ; 12$ & $2 \pm 0.5$ \\
\hline RPV & 35 & $10 \pm 2$ & $>20$ & $100 \% ; 12$ & $3 \pm 0.2$ \\
\hline LAA-LPV & 35 & $20 \pm 5$ & ND & $91 \% ; 11$ & $2 \pm 1$ \\
\hline LPV-RPV & 35 & $40 \pm 10$ & ND & $83 \% ; 10$ & $2.5 \pm 0.8$ \\
\hline
\end{tabular}

$L A A$, Left atrial appendage; $L P V$, left pulmonary vein; $R P V$, right pulmonary vein; $N D$, not determined.

Data are mean $\pm S D ; n=12$.

connecting lesions by grasping and pinching the targeted myocardial tissue with the jaws of the bipolar device. Two pinchings were needed to complete the full interconnecting lesion between the RPV and the LPV as well as between the LPV and the LAA. The time required for delivering each lesion ranged from 7 to 55 seconds (Table 1). The total procedure time was $14 \pm 6$ minutes. No complications were observed.

\section{Pacing Results}

Pacing thresholds before ablation were less than $1 \mathrm{~mA}$. Conduction could be assessed for RPV, LPV, and LAA lesions. A significant increase of the pacing threshold to supraphysiologic values was observed in all cases. The threshold was increased to more than $20 \mathrm{~mA}$ for all lesions except for 2 cases in which the atrium was captured at 15 $\mathrm{mA}$ from the isolated LAA (Table 1). Although the device indicated an impedance leveling and the lesions appeared transmural on postmortem analysis, the presence of micro gaps of nontransmurality in the thick and trabeculated LAA cannot be excluded and might explain the lower pacing threshold. Moreover, the TTC staining that was adopted does have limitations in the evaluation of postmortem transmurality as demonstrated in previous studies. ${ }^{9}$

\section{Postmortem Analysis}

No damage to surrounding structures in the mediastinum was observed. Gross examination of the LA and pulmonary veins revealed no evidence of tearing, barotrauma, or thrombus formation. Lesion analysis after TTC staining showed uniform transmural lesions in most instances (Table 1). LPV, RPV, and LAA lesions were transmural along their entire course in all cases (Figure 4). Transmurality was not complete along the entire course of the ablating lesion in 1 LAA-LPV and in 2 LPV-RPV interconnecting lesions. However, it should be pointed out that the entire lesion was categorized as nontransmural whenever any gap not completely transmural was found. The transmurality gaps were documented at the junction of the interconnecting lesion with either the LPV or the RPV lesion and were probably of technical nature, due to variable amounts of myocardial tissue that was actually pinched into the jaws of the device. As a matter of fact, access to the relevant areas and the positioning of the device was sometimes difficult and might have impaired the achievement of transmurality. We believe that technical limitations prevented us from getting a proper bite of tissue when the pinch technique was adopted. The problem might be surmountable by applying additional lesions or by increasing the amount of RF power to perform the interconnecting lesions. The width of ablating lesions ranged from 1 to $5 \mathrm{~mm}$ and tended to be larger on thick and trabeculated atrial tissue (ie, LAA) (Table 1).

\section{Intracardiac Echocardiography}

In each pig, the Acunav catheter was advanced via the right femoral vein into the RA using tip deflectability without fluoroscopic guidance. LA structures and pulmonary ventricle imaging were obtained as well as left ventricle longaxis and short-axis views.

Indexes of global LA and left ventricular systolic function, such as TMV and LVEF, were not affected significantly by LA ablation. No significant changes were observed in LPV and RPV diameter and flow, although the absence of acute pulmonary vein stenosis or blood flow acceleration does not exclude the development of a stenosis in the longer term. Moreover, no WMA or thrombus formation was detectable acutely after ablation. Echocardiographic data are summarized in Table 2.

\section{Electromechanical Mapping}

High-density maps counting an average of 200 sampled points were recorded. Unipolar and bipolar endocardial potentials were recorded by the catheter electrode and were converted into 3D electrical maps where the voltage value was displayed on a graded color scale (Figure 5). Endocardial zones with low electrical activity (ie, bipolar voltage $<$ $1 \mathrm{mV}$ ) were assumed to be representative of abnormal electromechanical activity. A distinct ablation pattern was reproduced by the electrical maps as demonstrated in Figure 5. The earliest electrical activation was detected on the medial aspect of the LA in the region of the Bachmann bundle and spread to the lateral and inferior aspects of the atrial chamber. The global LA AT was $49.4 \pm 8.8$ milliseconds prior to ablation. Postablation, the electrical activation pattern was similar to preablation (ie, starting in the 


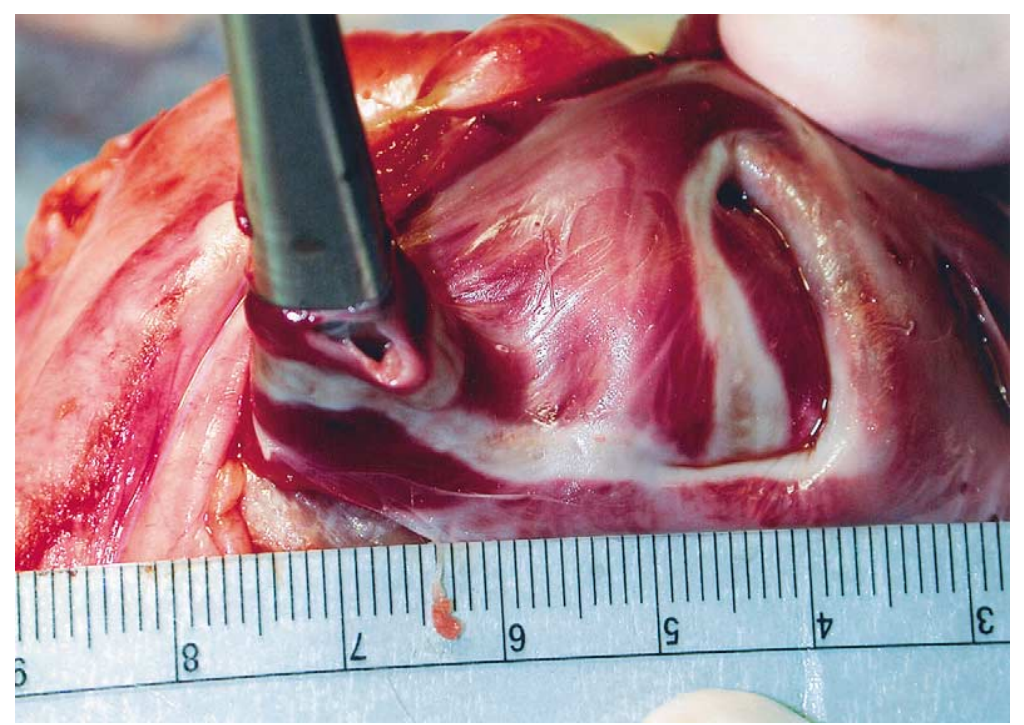

Figure 4. Endocardial view of the posterior LA showing transmural lesions following TTC staining.

Bachmann bundle region and spreading to the lateral and inferior LA) and the global LA AT was not significantly altered (59 \pm 9 milliseconds, $P=.18$ ). Although a large volume of LA myocardium was either ablated or electrically isolated (ie, endocardial points with bipolar voltage $<1 \mathrm{mV}$ increased from $12.38 \% \pm 9 \%$ to $29.5 \% \pm 9.4 \%, P=.01$ ), the ablation procedure was not associated with significant alterations in global LA mechanics (Table 3).

\section{Discussion}

The gold standard for the surgical treatment of $\mathrm{AF}$ is the maze III procedure developed by Cox and associates, ${ }^{10}$ in which multiple atrial incisions are made to direct the sinus impulse through a path or a maze to reach the atrioventricular node. Although the results reported by Cox were excellent, the standard maze III procedure has met poor physician and patient acceptance due to its invasiveness (sternotomy, $\mathrm{CPB}$ ) and significant morbidity. Moreover, the results achieved by Cox have not been reproduced by other groups and many centers that adopted the maze procedure did not achieve normal sinus rhythm conversion rates higher than $70 \%$ to $80 \%$.

Recent data suggest that an LA lesion pattern can achieve results comparable to the maze III procedure although extensive clinical data are needed. ${ }^{11-15}$ According to Haissaguerre and colleagues ${ }^{16}$ and Chen and colleagues, ${ }^{17}$ paroxysmal AF originates from focal ectopies arising from the pulmonary veins and resulting in simultaneous reentrant wavelets. Based on these findings and assuming that $\mathrm{AF}$ is a disorder of the LA, the performance of a left-sided ablation might be appropriate for treating paroxysmal and most cases of chronic AF. Percutaneous methods may be applied to the right atrium if needed. Consequently, innovative
TABLE 2. Echocardiographic results

\begin{tabular}{lccc}
\hline Variable & Preablation & Postablation & $\boldsymbol{P}$ value \\
\hline TMV (m/s) & $2.34 \pm 0.32$ & $2.64 \pm 0.3$ & .06 \\
MR & None & None & - \\
LVEF (\%) & $46.2 \pm 8.64$ & $46.6 \pm 7.8$ & .9 \\
WMA & None & None & - \\
LA thrombus & None & None & - \\
RPV diameter (mm) & $0.54 \pm 0.09$ & $0.52 \pm 0.08$ & .37 \\
LPV diameter (mm) & $0.7 \pm 0.12$ & $0.74 \pm 0.19$ & .37 \\
RPV flow & & & \\
P1 (m/s) & $0.94 \pm 0.09$ & $0.98 \pm 0.08$ & .17 \\
P2 (m/s) & $0.86 \pm 0.09$ & $0.84 \pm 0.13$ & .37 \\
A (m/s) & $0.34 \pm 0.11$ & $0.32 \pm 0.08$ & .74 \\
LPV flow & & & \\
P1 (m/s) & $1.22 \pm 0.21$ & $1.38 \pm 0.26$ & .3 \\
P2 (m/s) & $1.02 \pm 0.04$ & $1.04 \pm 0.06$ & .9 \\
A (m/s) & $0.36 \pm 0.13$ & $0.38 \pm 0.13$ & .77
\end{tabular}

$T M V$, Peak transmitral valve inflow; $M R$, mitral regurgitation; $L V E F$, left ventricular ejection fraction; $W M A$, wall motion abnormalities; $L A$, left atrium; $R P V$, right pulmonary vein; $L P V$, left pulmonary vein.

Data are mean $\pm \mathrm{SD} ; \mathrm{n}=5$.

approaches to AF ablation have been developed, consisting of the confinement of the lesions to the LA, the substitution of RF or other source of energy for atriotomies, and the development of a closed heart epicardial off-pump approach.

Our objective was to develop an effective technique to deliver the ablating energy from the epicardial surface because: (1) epicardial ablation might be safer as the energy is directed into the atrial chamber rather than outward into adjacent mediastinal structures; (2) CPB can be avoided; (3) access to the relevant structures is easier than with catheterbased techniques. 


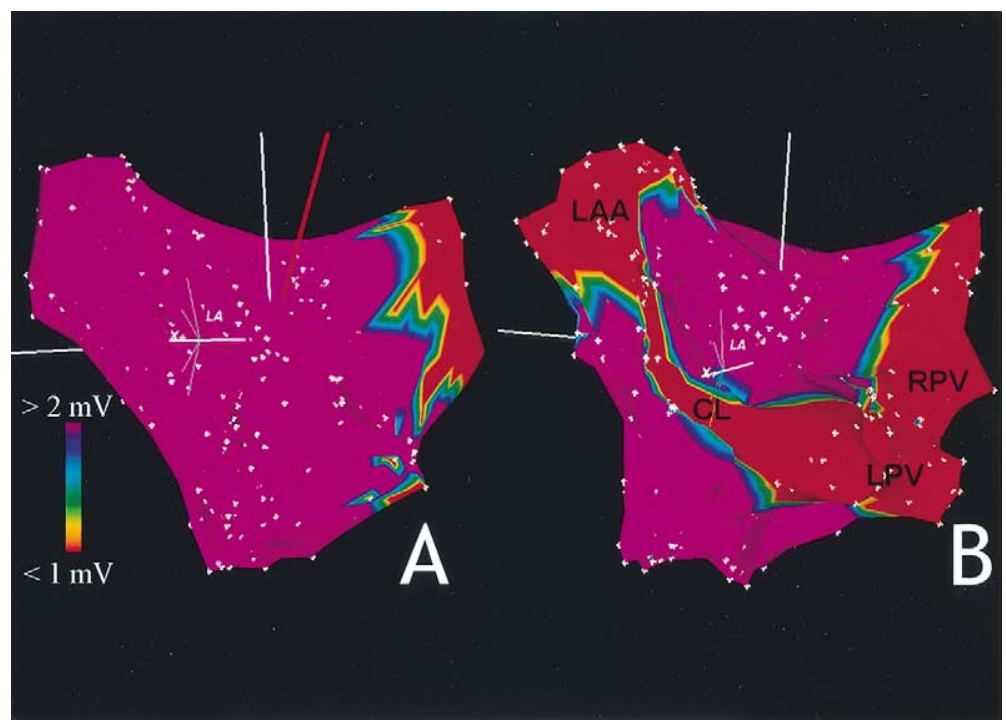

Figure 5. Color-coded electrical mapping using the NOGAJ system: (A) preablation; (B) postablation. $L A A$, left atrial appendage ablation; $C L$, connecting lesion; $L P V$, left pulmonary vein ablation; $R P V$, Right pulmonary vein ablation.

TABLE 3. NOGA results

\begin{tabular}{lccc}
\hline Variable & Preablation & Postablation & $\boldsymbol{P}$ value \\
\hline LA EDV (mL) & $44 \pm 9.9$ & $42.3 \pm 9$ & .42 \\
LA ESV (mL) & $34.55 \pm 8.9$ & $34.25 \pm 6.8$ & .79 \\
LA EF (\%) & $0.19 \pm 0.1$ & $0.17 \pm 0.08$ & .47 \\
Global LA AT (ms) & $49.4 \pm 8.8$ & $58.8 \pm 9$ & .18 \\
Bipolar voltage $<1 \mathrm{mV} \mathrm{( \% )}$ & $12.38 \pm 9$ & $29.5 \pm 9.5$ & .01 \\
\hline
\end{tabular}

$L A$, Left atrium; $E D V$, end-diastolic volume; $E S V$, end-systolic volume; $E F$, ejection fraction; $A T$, activation time.

Data are mean $\pm S D ; n=5$.

The application of RF energy for atrial ablation may be either unipolar or bipolar, irrigated or nonirrigated. In unipolar RF, the energy is applied between the tip of the electrode and a grounding plate. ${ }^{3,13,14}$ Bipolar RF energy has recently been adopted in an experimental study to achieve transmural lesions on the beating heart. ${ }^{18} \mathrm{We}$ developed a bipolar device in which the energy is delivered between 2 irrigated electrodes on the opposite jaws of a clamping instrument. The bipolar mode was expected to enhance the effectiveness of RF energy delivery to the targeted atrial tissue while normal saline irrigation was adopted to delay the impedance rise and produce deeper and transmural lesions. ${ }^{19,20}$ The study conducted by Nakagawa and colleagues ${ }^{19}$ showed that saline irrigation of RF electrodes was associated with a reduction of the impedance at the surface, allowing the energy to cross deeper into the tissue and the surface cooling preventing any charring or dessication. However, excellent transmurality results and no charring were also achieved when bipolar energy was ap- plied without irrigation and using a different lesion assessment algorithm based on temperature control. ${ }^{18}$

A major concern when performing an epicardial ablation is the accomplishment of transmural lesions to ensure conduction block. Transmurality may be impaired by the thickness of the epicardium, which can be wrapped with fat tissue, and the convective cooling of circulating blood. No investigation comparing irrigated versus nonirrigated or impedance versus temperature control has been published. In this study, we demonstrated that beating heart epicardial LA ablation was feasible and reproducible; the irrigated bipolar RF device proved to be very effective in producing transmural lesions and conduction block without signs of charring or barotrauma. Moreover, the procedure could be performed safely and under direct vision, avoiding any damage to surrounding cardiac or mediastinal structures. The ablation pattern that we adopted was described in a recent experimental study by Kress and associates. ${ }^{11}$ It included a bilateral pulmonary vein isolation that spared part of the posterior LA wall, a connecting lesion between the 2 pulmonary veins, the electrical isolation of the base of the LAA, and a connecting lesion between the LPV and the LAA lesions. The connecting lesion from the mitral valve to the pulmonary veins was avoided because it may injure the circumflex coronary artery and its utility is debatable..$^{21,22}$ Our study shows that this pattern, which was highly successful in a canine AF model, ${ }^{11}$ is feasible and safe when performed epicardially and off-pump. The development of a minimally invasive approach might further expand the indications for lone AF surgical treatment. 
The objective of surgical ablation is to restore normal sinus rhythm, atrioventricular synchrony, and atrial contraction. It has been demonstrated that the atrial function is depressed following electrical, pharmacological, and spontaneous cardioversion of $\mathrm{AF}^{23,24} \mathrm{AF}$ is associated with the electrical, contractile, and structural remodeling of the atria leading to reduced atrial function after cardioversion. ${ }^{25} \mathrm{Al}-$ though several studies have reported the presence of atrial contractions after the maze procedure, little is known about the atrial transport function. Some studies are consistent with a decrease in the LA transport function after the maze procedure in paroxysmal AF (ie, no associated heart disease). ${ }^{26,27}$ In addition to the effects of atrial remodeling, further impairment of atrial function may be due to the extensive cut and sew technique of the maze procedure, which leads to atrial mass reduction, interruption of atrial microcirculation, and tissue scarring. ${ }^{27-29}$

In this study we hypothesized that epicardial LA ablation per se using a new bipolar RF device would not result in significant electromechanical consequences upon normal LA physiology. Intracardiac echocardiography and mapping using the NOGA system were used to assess the LA electromechanical properties prior to and after ablation. We found that although a significant amount of atrial myocardium was either ablated or electrically isolated (ie, $17.12 \%$ $\pm 9 \%$ ), the global LA activation time and pattern, the LA volumes and ejection fraction, the pulmonary vein diameter and flows, and the TMV and LVEF were not significantly altered. Moreover, no LA thrombus formation, mitral valve regurgitation, or wall motion abnormality was detected acutely following LA ablation.

\section{Study Limitations}

The experimental model is intended solely to study the feasibility and safety of beating heart epicardial ablation using a bipolar RF device. The electromechanical properties were studied during normal sinus rhythm and therefore reflect the effects of epicardial RF ablation per se upon LA function and might not be applicable to the fibrillating LA. The assessment was performed acutely (ie, 1 hour after ablation) and further studies are warranted to exclude a progressive decline of LA function and the development of pulmonary vein stenosis in the longer term.

\section{References}

1. Cox JL, Schuessler RB, D'Agostino HJ, et al. The surgical treatment of atrial fibrillation: III. Development of a definite surgical procedure. J Thorac Cardiovasc Surg. 1991;101:569-83.

2. Cox JL, Ad N. New surgical and catheter-based modifications of the Maze procedure. Semin Thorac Cardiovasc Surg. 2000;12:68-73.

3. Sie H, Beukema W, Ramdat Misier AR, et al. Radiofrequency modified maze in patients with atrial fibrillation undergoing concomitant cardiac surgery. J Thorac Cardiovasc Surg. 2001;122:249-56.

4. Williams MR, Argenziano M, Oz MC. Microwave ablation for surgical treatment of atrial fibrillation. Semin Thorac Cardiovasc Surg. 2002;14:232-7.
5. Maessen JG, Nijs JF, Smeets JL, Vainer J, Mochtar B. Beating-heart surgical treatment of atrial fibrillation. Ann Thorac Surg. 2002;74: 1307-11.

6. Castello R, Pearson AC, Lenzen P, Labovitz AJ. Evaluation of pulmonary venous flow by transesophageal echocardiography in subjects with abnormal heart: comparison with transthoracic echocardiography. J Am Coll Cardiol. 1991;18:65-71.

7. Packer DL, Stevens CL, Curley MG, et al. Intracardiac phased-array imaging. Methods and initial clinical experience with high resolution, under blood visualization: initial experience with intracardiac phasedarray ultrasound. J Am Coll Cardiol. 2002;39:509-16.

8. Gepstein L, Hayam G, Shpun S, Ben-Haim SA. Hemodynamic evaluation of the heart with a nonfluoroscopic electromechanical mapping technique. Circulation. 1997;96:3672-80.

9. Adegboyega PA, Adesokan A, Haque AK. Sensitivity and specificity of triphenyl tetrazolium chloride in the gross diagnosis of acute myocardial infarcts. Arch Pathol Lab Med. 1997;121:1063-8.

10. Cox JL, Jaquiss R, Schuessler R, Boineau J. Modification of the maze procedure for atrial flutter and atrial fibrillation. II. Surgical technique of the maze III procedure. J Thorac Cardiovasc Surg. 1995;110:48595 .

11. Kress DC, Krum D, Chekanov V, et al. Validation of a left atrial lesion pattern for intraoperative ablation of atrial fibrillation. Ann Thorac Surg. 2002;73:1160-8.

12. Sueda $T$, Nagata $H$, Orihashi $K$, et al. Efficacy of a simple left atrial procedure for chronic atrial fibrillation in mitral valve operations. Ann Thorac Surg. 1997;63:1070-5.

13. Kottkamp H, Hindricks G, Autschbach R, et al. Specific linear left atrial lesions in atrial fibrillation. Intraoperative radiofrequency ablation using minimally invasive surgical techniques. J Am Coll Cardiol. 2002;40:475-80.

14. Melo J, Adragao P, Neves J, et al. Endocardial and epicardial radiofrequency ablation in the treatment of atrial fibrillation with a new intra-operative device. Eur J Cardiothorac Surg. 2000;18:182-6.

15. Deneke T, Khargi K, Grewe PH, et al. Left atrial versus bi-atrial maze operation using intraoperatively cooled-tip radiofrequency ablation in patients undergoing open-heart surgery. J Am Coll Cardiol. 2002;39: 1644-50.

16. Haissaguerre M, Jais $\mathrm{P}$, Shah DC, et al. Spontaneous initiation of atrial fibrillation by ectopic beats originating in the pulmonary veins. $N$ Engl J Med. 1998;339:659-66.

17. Chen SA, Hsieh MH, Tai CT, et al. Initiation of atrial fibrillation by ectopic beats originating in the pulmonary veins: electrophysiological characteristics, pharmacological responses, and effects of radiofrequency ablation. Circulation. 1999;100:1879-86.

18. Prasad SM, Maniar HS, Schuessler RB, Damiano RJ. Chronic transmural atrial ablation by using bipolar radiofrequency energy on the beating heart. J Thorac Cardiovasc Surg. 2002;124:708-13.

19. Nakagawa H, Yamanashi WS, Pitha JV, et al. Comparison of in vivo tissue temperature profile and lesion geometry for radiofrequency ablation with a saline-irrigated electrode versus temperature control in a canine thigh muscle preparation. Circulation. 1995;91:2264-73.

20. Demazumder D, Mirotznik MS, Schwartzman D. Biophysics of radiofrequency ablation using an irrigated electrode. J Interv Card Electrophysiol. 2001;5:377-89.

21. Berreklouw E, Bracke F, Meijei A, Peels KH, Relik D. Cardiogenic shock due to coronary narrowings one day after a Maze III procedure. Ann Thorac Surg. 1999;68:1065-6.

22. Benussi S, Pappone C, Nascimbene S, et al. A simple way to treat chronic atrial fibrillation during mitral valve surgery: the epicardial radiofrequency approach. Eur J Cardiothorac Surg. 2000;17:524-9.

23. Allessie M, Ausma J, Schotten U. Electrical, contractile and structural remodelling during atrial fibrillation. Cardiovasc Res. 2002;54:230-46.

24. Harjai KJ, Mobarek SK, Cheirif J, Boulos LM, Murgo JP, Abi-Samra F. Clinical variables affecting recovery of left atrial mechanical function after cardioversion from atrial fibrillation. J Am Coll Cardiol. 1997;30:481-6.

25. Grimm RA, Leung DY, Black IW, Stewart WJ, Thomas JD, Klein AL. Left atrial appendage stunning after spontaneous conversion of atrial fibrillation demonstrated by transesophageal Doppler echocardiography. Am Heart J. 1995;130:174-6. 
26. Lonnerholm S, Blomstrom P, Nilsson L, Blomstrom-Lundqvist C. Atrial size and transport function after the Maze III procedure for paroxysmal atrial fibrillation. Ann Thorac Surg. 2002;73:107-11.

27. Jessurun ER, Van Hemel NM, Defuaw J, et al. Results of Maze surgery for lone paroxysmal atrial fibrillation. Circulation. 2000;101: 1559-67.
28. Tsui S, Grace AA, Ludman PF. Maze 3 for atrial fibrillation: two cuts too few? Pacing Clin Electrophysiol. 1994;17:2163-6.

29. Nitta T, Lee R, Schuessler RB, Boineau JP, Cox JL. Radial approach: a new concept in surgical treatment for atrial fibrillation. 1. Concept, anatomic and physiologic bases and development of a procedure. Ann Thorac Surg. 1999;67:27-35. 